\title{
Switching of a bi-stable diverter valve with synthetic jet actuators
}

\author{
Nicholas D. Martin ${ }^{1}$ and Michael Bottomley ${ }^{1}$ and Alan Packwood ${ }^{2}$ \\ University of Surrey, Guildford, Surrey, GU2 $7 X H$
}

\section{Introduction}

IVERTER valves for the control of internal fluid flows have a number of engineering applications. Classical designs, which use numerous mechanical parts, can have the disadvantages of potential unreliability in adverse environmental conditions, and high power consumption. There has therefore been research interest in the development of fluidic oscillators that use fewer, lower mass moving parts. These systems rely on fluidic amplification, whereby a control flow acts on the primary flow requiring switching. Generally, the control (flow) is at an order of magnitude lower momentum than the primary.

Bi-stable fluidic oscillators have been investigated in a number of experiments ${ }^{1-7}$. In the work of Tesar ${ }^{8}$, the control ports that form the wall normal jet were connected together with a tube, creating a feedback loop. This creates a self-sustaining oscillation between the two channels that can be controlled with the loop length or the oncoming flow rate. Recently, these oscillatory flow devices have been used for flow separation control on an asymmetric bluff body ${ }^{9}$. Fluidic oscillators that use the concept of control authority provided by an piezoelectric element have been investigated by others ${ }^{10,11}$. The advantages of using a piezo element in the valve is the relative ease of variation in the duty cycle of the oscillation. This is particularity desirable in applications such as flow separation control ${ }^{12}$, where the precise flow conditions of the valve may change, not be known beforehand, or where the flow control effect is a strong function of the frequency ${ }^{13-15}$. The aim of this study is to give an overview of an alternative mechanism to control a bi-stable diverter valve, using synthetic jet (SJ) actuators. A SJ has a zero net mass flux, but a net momentum flux across the system boundary ${ }^{16}$. They are compact, low power devices, which make them ideal for this application.

\footnotetext{
${ }^{1}$ Research Student, Department of Mechanical and Engineering Sciences, University of Surrey, GU2 7XH.

${ }^{2}$ Senior Tutor, Department of Mechanical and Engineering Sciences, University of Surrey, GU2 7XH.
} 


\section{Experimental setup}

The experiments were conducted in air, at the EnFlo laboratory at the University of Surrey. The primary flow was supplied by a compressed air supply that was controlled by a closed-loop system of a Bronkhurst High Tech EL-FLOW mass flow meter, and an in-house, LabVIEW based instrumentation control and data acquisition system.

\section{A. Particle image velocimetry}

PIV measurements of the instantaneous velocity field of both outlets were made in order calculate the volumetric flow rate of each port. A $0.19 \mathrm{~m}^{2}$ measurement window was illuminated by dual $50 \mathrm{~mJ} \mathrm{Nd}: Y A G$ lasers expanded into a light sheet of a $\sim 1 \mathrm{~mm}$ thickness. The plane of the light sheet was perpendicular to the exit face of the switch and at half the depth of the channels. The flow was seeded flow was seeded with $\sim 1 \mu$ m diameter olive oil tracer particles. Images were taken with a TSI Powerview 4MP CCD camera, at $7.25 \mathrm{~Hz}$, and 50 pairs were collected to

ensure the mean velocity field had statistically converged. TSI Insight 4G software was used for the image to vector processing. Images were divided into an equally spaced grid of $\sim 1.5 \mathrm{~mm}^{2}$ (16x16 pixels) and a FFT cross-correlation algorithm was used to obtain the velocity field.

\section{B. Constant temperature hot wire anemometry}

The CTA used was a TSI 1750 model connected to a modified hot-wire probe, built in-house. A $0.9 \mathrm{~mm}$ length of $5 \mu \mathrm{m}$ diameter platinum coated tungsten wire was welded between the two prongs. The hot wire was used to measure the velocity magnitude of the diverter valve at the exit of each channel as well as for the SJ. The hot wire was calibrated in a closed return wind tunnel, against a pitot static tube up to $40 \mathrm{~m} / \mathrm{s}$. The tunnel had previously been measured to have a streamwise turbulence intensity of $\sim 0.2 \%$. The sampling rate was set at $50,000 \mathrm{~Hz}$, more than one order of magnitude higher than the frequency of the SJ.

\section{Design of the fluidic switch and synthetic jet}

\section{A. Fluidic switch}

The design of the valve was adapted from that previously designed by Tesar ${ }^{6}$. The dimensions were scaled up and some modifications to the geometry were incorporated to allow the additional SJ components to be incorporated. The dimensions of the valve, as a function of the inlet nozzle width, $b$ can be seen in Figure. 1. The valve has a 
nozzle width of $b=12 \mathrm{~mm}$. The design is planar, with control ports spanning the height of the nozzle. All ports have rectangular cross-sections, and the height of the valve, $h=1.66 b$.

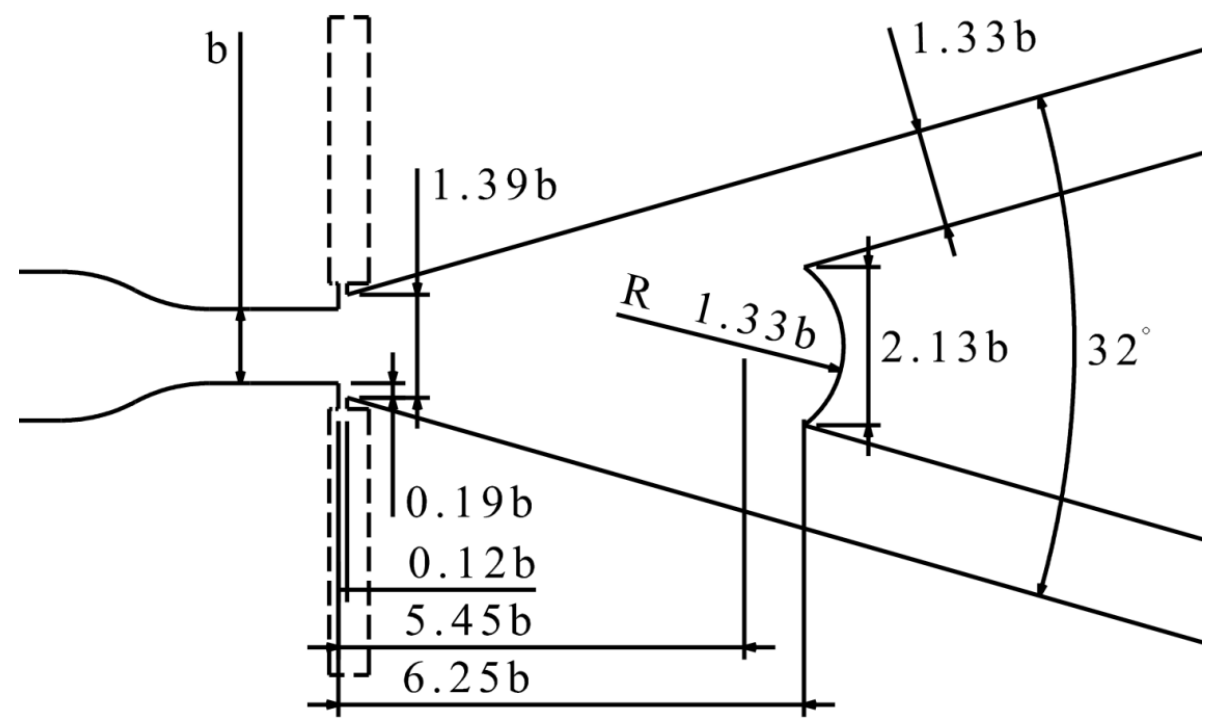

Figure 1. Schematic of the fluidic switch. Dashed lines represent the synthetic jet cavity

Surface flow visualization was conducted in order to understand both how the large scale structures effect the control of the switch, and for comparison with the aforementioned literature ${ }^{6}$. Still images were taken in a darkened room with a UV light to ensure ideal image contrast. Videos were also taken of the switch so that the direction of the vortex rotation could be established.
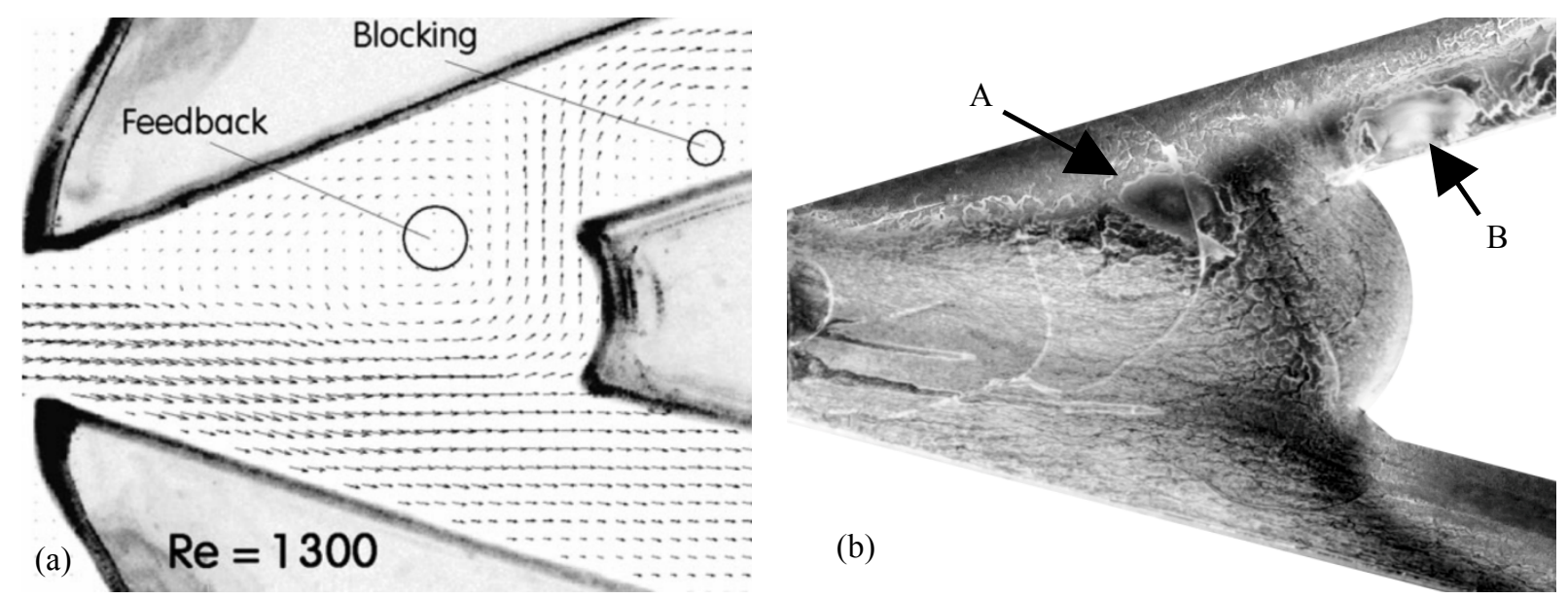

Figure 2. Comparison of PIV (a) from ${ }^{3}$ with surface flow visualization (b) at $R e=1557$ 
Figure 2 (a) shows a PIV vector field of a fluidic switch from ${ }^{3}$, in comparison to the flow visualization in image (b) of the fluidic switch used in this investigation. Both are at similar Reynolds numbers, and show some clear similarities. The two main rotating structures referred to as 'Feedback' and 'Blocking' ${ }^{6}$ in Figure 2 (a), are evident in (b), and the direction of rotation identified from videos was seen to compare to that evident in the vector field.

PIV data was taken at 3 different inlet mass flow rates. The volumetric flow rate was approximated by integration of the measured outlet velocity, and extrapolation of the 2D field across the full outlet height. The total flow rate was taken to be the sum of both ports, (the 'off' and 'on' cases were recorded in separate measurements), with no losses through the valve from the inlet. From this, the nozzle velocity $\left(U_{n}\right)$ and therefore Reynolds number $R e=U_{n} h / v$, could be estimated. The characteristic length was taken to be the width of the nozzle $b$. The results are shown in Table 1, where an efficiency ratio $=\eta$ is given for each port and is defined by:

$$
\eta=\frac{Q}{Q_{i}}
$$

where $Q_{i}$ is the total volumetric flow rate through the inlet nozzle, and $Q$ the flow at the outlet, when in the 'off' condition. A minimum of $79 \%$ of the mass flow would be directed to the 'on' port for any case tested. This demonstrates similar performance to other examples of such design ${ }^{6,8,17}$. The results in Table 1 show a slight asymmetry in the results between $\eta_{l}$ and $\eta_{2}$, deemed due to manufacturing tolerances on the valve.

Table 1. Mass flow rates calculated from PIV

\begin{tabular}{cccc}
\hline \hline$Q\left(\mathrm{~m}^{3} / \mathrm{s}\right)$ & $\eta_{1}$ & $\eta_{2}$ & $R e$ \\
\hline 0.001403 & 0.216 & 0.150 & 565 \\
0.002523 & 0.177 & 0.137 & 1017 \\
0.003862 & 0.178 & 0.162 & 1557 \\
\hline \hline
\end{tabular}

\section{B. Synthetic Jet Design}

The design of the SJ actuator is based upon a design used previously for flow control investigations at the University of Surrey ${ }^{18}$. The disc was a commercially available PUI Audio AB4113B bi-morph piezoelectric 'buzzer' disc, of $41 \mathrm{~mm}$ diameter. This particular disc was selected as the resonant frequency of the disc is close to that of the cavity, which acts like a Helmholtz resonator. It has been shown to work with similar cavities in previous studies $^{18}$.

The jet cavity is made using a Nylon-12 polyamide, and a commercial selective laser sintering (SLS) rapid prototyping machine which enables complex, convex geometries to be manufactured with relative ease and low cost. Figure 3 shows the frequency response of the assembled actuator for a sinusoidal waveform of $80 \mathrm{~V}$ peak-to- 
peak. The piezo discs were driven using a Tektronix AFG3022B two channel function generator. This was interfaced with the computer used for data acquisition so that the frequency and sampling rate could be set in order that a predetermined number of cycles and samples per cycles were acquired at each frequency. The signal was amplified using a commercially available high quality audio amplifier. The velocity of the jet was measured at the exit plane of the orifice using the hot wire described in section II B. The measurements were taken with the wire orientated parallel to the orifice slot exit plane on its centerline at approximately one slot width $w$ above the exit plane, where $w=0.12 b$. The velocity was then phase averaged over 30 cycles for each frequency. Figure 3 shows the peak jet velocity magnitude $U_{j}$. The two peaks in the frequency response are present at approximately $630 \mathrm{~Hz}$ and $1000 \mathrm{~Hz}$ respectively. These peaks are related (but not equal) to the disc resonant frequency $f_{D}$ and the cavity Helmholtz frequency $f_{H}{ }^{19}$. They occur because the disc and cavity are coupled, which gives rise to a fourth order transfer function between the input voltage and output velocity of the SJ. The system will therefore have two resonant frequencies. Gallas ${ }^{20}$ describes the relationship between these frequencies:

$$
\psi^{2}-\left[f_{D}^{2}\left(1-C_{d} / C_{c}\right)+f_{H}^{2}\right] \psi+f_{D}^{2} f_{H}^{2}=0
$$

Where $C_{d}$ and $C_{c}$ are the disc and cavity acoustic compliance and the two roots of $\psi$ are the square of the two frequency peaks. In the limit as $C_{d} / C_{c} \rightarrow 0$ the first peak becomes equal to the Helmholtz frequency and the second peak to the disc frequency. For this case the Helmholtz frequency is calculated to be $614 \mathrm{~Hz}$, which is close to the experimentally measured value of approximately $630 \mathrm{~Hz}$. From Equation (2) the second peak is calculated as $1320 \mathrm{~Hz}$. However, experimentally the second peak is clearly much closer to $1000 \mathrm{~Hz}$. This could be related to the sensitivity of piezo discs to their boundary conditions, which has a direct effect on the disc resonant frequency ${ }^{21}$. 


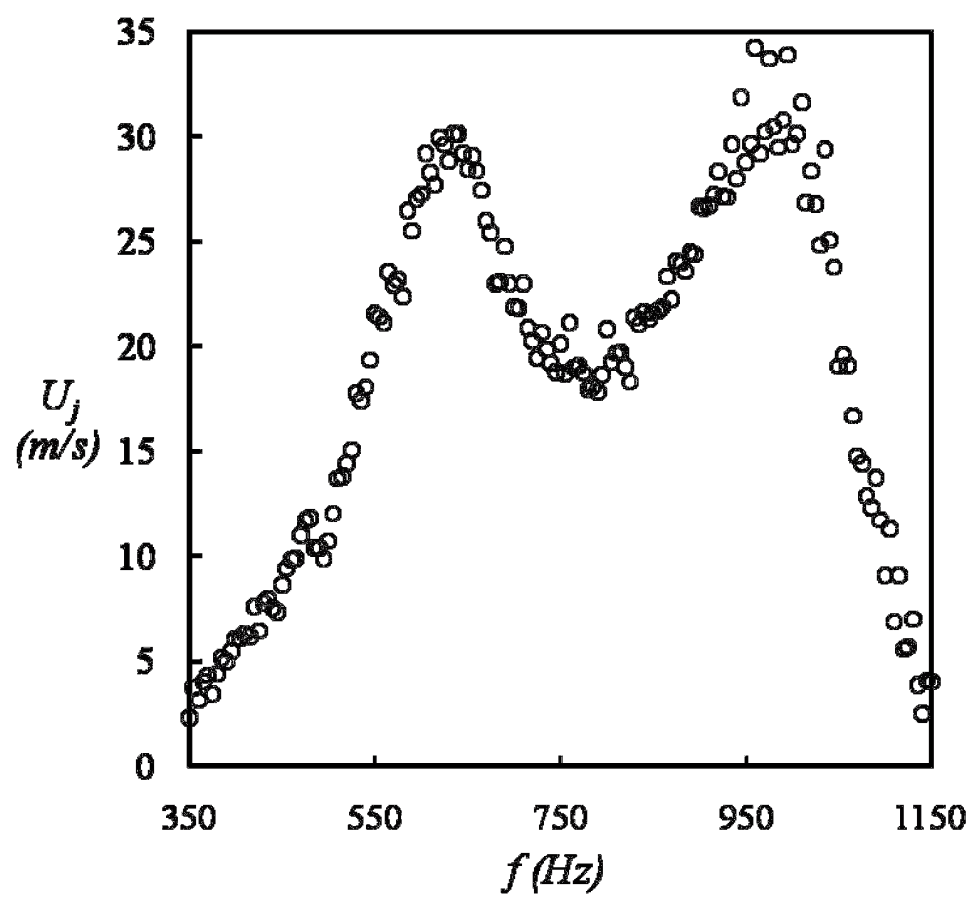

Figure 3. Frequency response of the synthetic jet actuator at $630 \mathrm{~Hz}$

\section{Results}

\section{A. Synthetic Jet Switching}

Initial testing confirmed that the SJs were able to control the switching of the diverter valve, with the jet operating at a frequency of $630 \mathrm{~Hz}$, corresponding to the first peak from Figure 3. With this established, tests involved looking at the transient nature of the switching process. The hot wire probe was placed in the 'off' channel and the data acquisition software triggered to start taking data as the SJ was turned on. To retain the symmetrical nature of the valve during measurements, a dummy hot-wire probe of the same specification was positioned into the opposite outlet port. This was in order to avoid introducing unequal back pressures to the ports, which could alter the switching characteristics of the jet.

The CTA data was taken as described in section II B and the results are displayed in Figure 4 (a) for $R e=824$. Immediately after the jet is switched on, oscillations in the flow at the same frequency are detected by the CTA. The velocity gradually builds over a number of jet cycles, until after approximately 60 cycles where the velocity levels off after the switching has completed. Once switched the oscillations of the SJ are still present in the velocity time trace. 

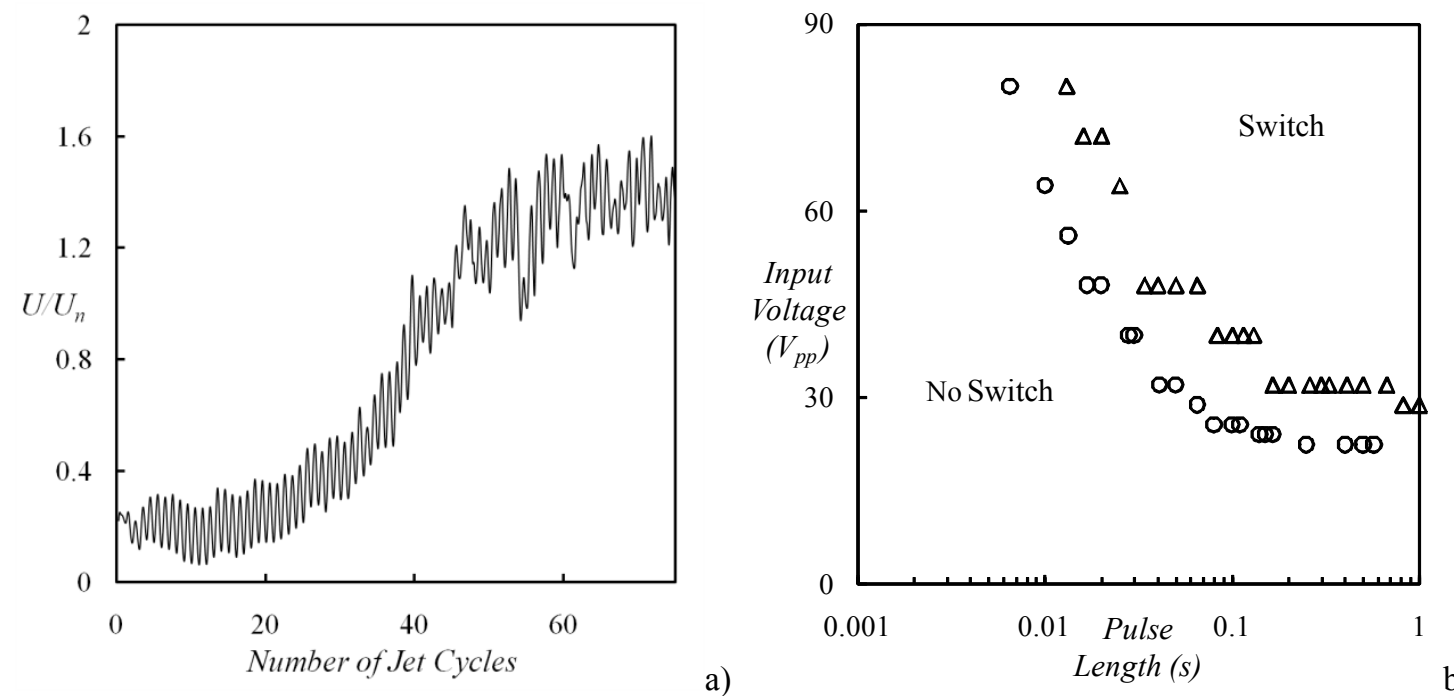

b)

Figure 4. (a) Dimensionless velocity at the exit of the channel as a function of synthetic jet cycles, at a jet frequency of $630 \mathrm{~Hz}$, (b) Peak to peak voltage applied to the SJ with the SJ pulse length; ○, Re = 565; $\triangle$, $\operatorname{Re}=1557$

To assess the switching effect of the SJ, the function generator was used to create a 'burst mode' signal of the jet at the same carrier signal frequency of $630 \mathrm{~Hz}$ used previously. The pulse length was varied, as well as the peak-topeak amplitude of the input voltage. The results are displayed in Figure 4 (b) for the highest and lowest Reynolds numbers tested, 565 and 1557 . Here the data points represent the input voltage, and pulse length required to guarantee that the diverter valve will switch. To the left of or below these curves, the switch will not be able to operate, whereas to the right of or above the curve, switching is guaranteed. The amplitude and pulse length of the jet appear to asymptote as each value decreases. There exists a threshold limit to the amplitude or pulse duration whereby switching will not occur below this limit. Of particular interest is that despite the velocity magnitude of the first and second resonant frequencies of the SJ being similar, it is only when operating at $630 \mathrm{~Hz}$ that switching was found to be possible. This indicates that the mechanism behind how the SJ perturbs the flow to switch from one channel to the other may not only be based upon the momentum imparted by the jet. Investigating why this is will be the focus of future work, as optimizing this frequency may allow switching at higher flow rates.

\section{B. Oscillatory control}

Oscillatory control was investigated by activating both SJs on separate duty cycles so the flow would periodically switch from one channel to the next. This was achieved by modulating the amplitude of the $630 \mathrm{~Hz}$ carrier wave in a continuous modulation mode. Each SJ signal was generated from separate channels on the function 
generator, and synchronized to be in opposing points of the square-wave phase. The switching frequency is the selected frequency of the continually modulated signal.

Initial tests showed the technique proved successful at switching the flow at discrete frequencies, which could be dynamically changed during tests. Figure 5 shows the results of altering the modulation signal frequency for two Reynolds numbers. Hot wire data was collected for 4 seconds, in the manner discussed previously, representing several complete switching cycles for all tests. Results were then phased averaged by the modulation frequency.

The results clearly demonstrate that the velocity in the port was able to be varied in a sinusoidal manner for all frequencies tested. Both Reynolds numbers show the same trends with higher frequencies delivering less peak to peak amplitude.
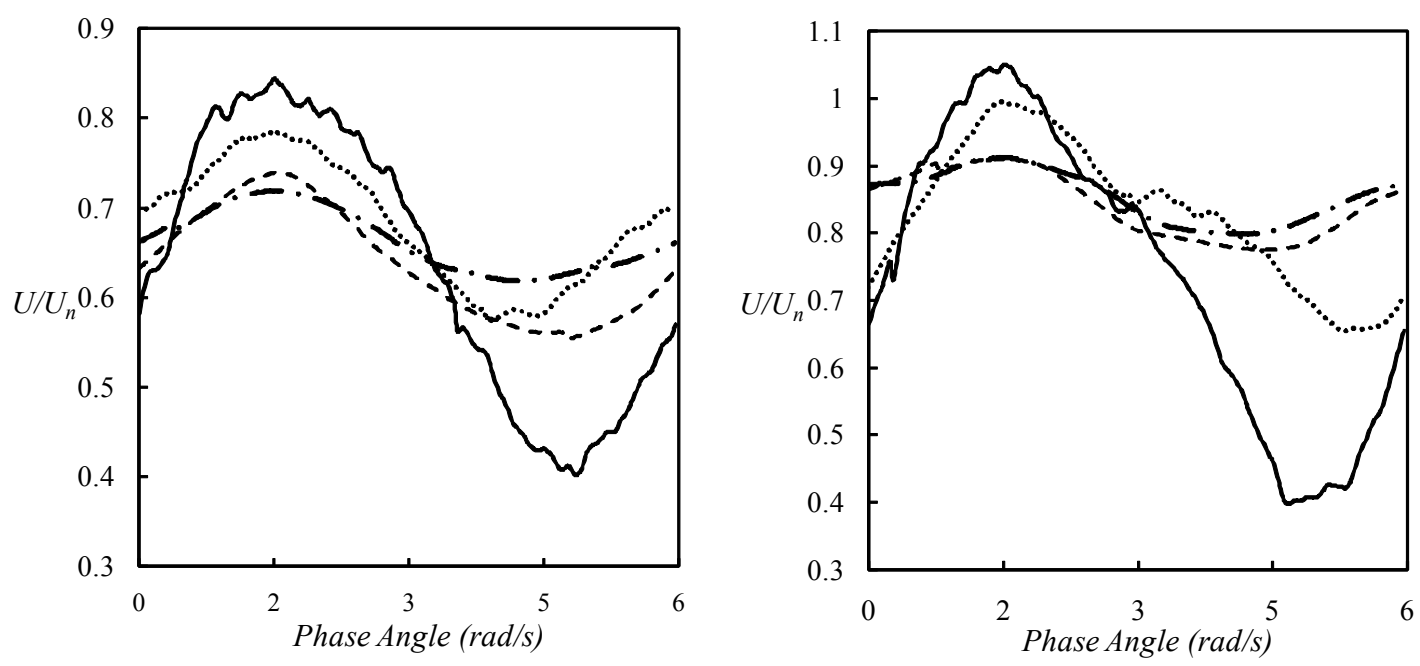

a)

Figure 5. Phase averaged dimensionless velocity for $R e=565$ (a) and $R e=824$ (b)

$$
-, \mathrm{f}_{\mathrm{c}}=5 \mathrm{~Hz} ; \bullet, \mathrm{f}_{\mathrm{c}}=10 \mathrm{~Hz} ;--, \mathrm{f}_{\mathrm{c}}=20 \mathrm{~Hz} ;-\bullet-, \mathrm{f}_{\mathrm{c}}=25 \mathrm{~Hz}
$$

This result can be understood by considering the effect of decreasing the duty cycle length of the actuator signal. If the modulation frequency is too high for a given Reynolds number, the flow will not reach a complete state transition. This limitation is not overcome just by increasing the amplitude of the SJ. There is a limit to the time period at which the flow can switch, determined by the geometry of the switch and the flow rate through the nozzle ${ }^{8}$.

The ratio of maximum to minimum velocity $\left(V_{r}\right)$ from the phase averaged results is presented as a function of the Strouhal number $(S t)$, defined as a function of the frequency of the modulation signal $\left(f_{m}\right)$, giving: 


$$
S t=\frac{f_{m} b}{U_{n}}
$$

Figure 6 shows that the results asymptote towards unity as the Strouhal number increases. Achieving larger velocity ratios for higher frequencies is a clear aim in order to improve the operating window of these devices. As that for different Reynolds numbers there exists a limit to the achievable Strouhal number for a given velocity ratio, it indicates there is a limit to the frequency that the jet enables switching, for a given geometry and nozzle velocity. Therefore, to increase the frequency, the jet must be able to switch the flow in the same period at higher nozzle velocities. This would suggest the requirement for more powerful SJs that can command authority over higher nozzle velocities.

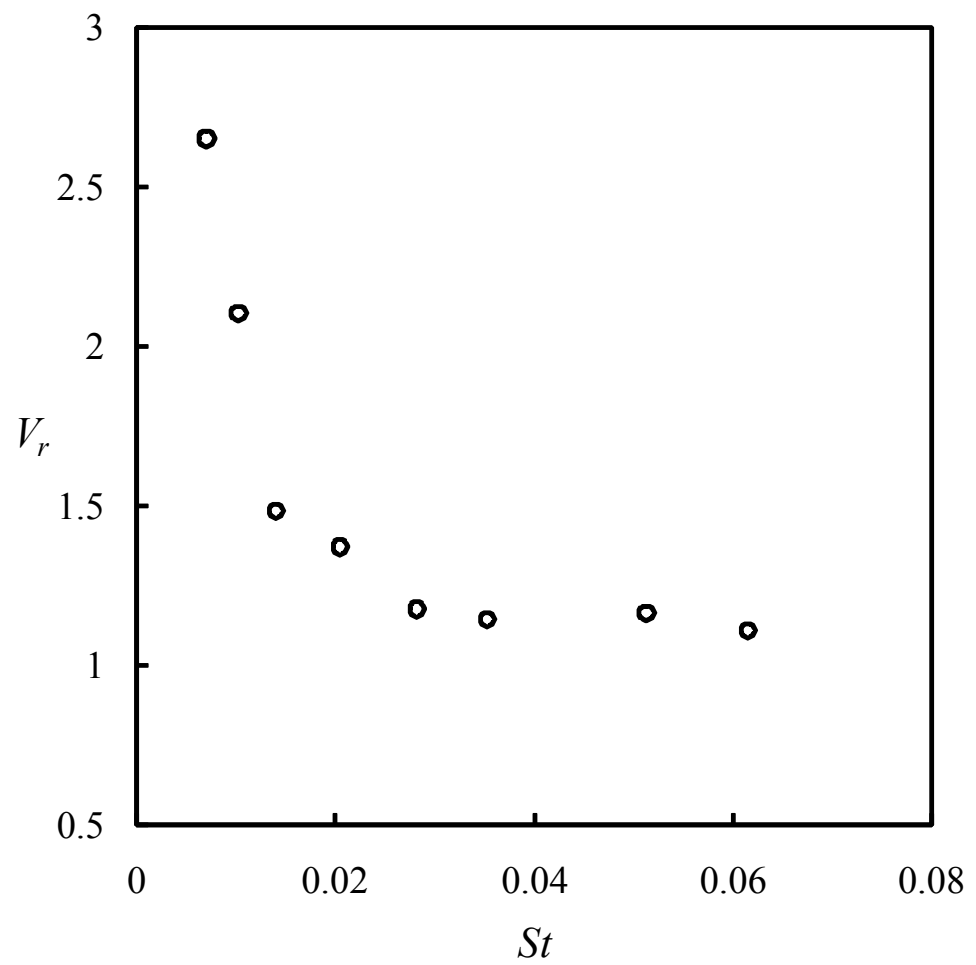

Figure $6 V_{r}$ as a function of Strouhal Number

\section{Conclusion}

Presented here is the development of a synthetic jet actuator controlled bi-stable fluidic diverter valve.

The valve has been characterized for a range operating conditions, where it has been demonstrated that an SJ can provide control authority, independently of conditions of the inlet flow, within the range tested. Further experiments 
with measurement techniques such as PIV in the actuation area of the switch would be of interest to aid further understanding of the initial results seen to date. Understanding of why switching success depends on jet frequency for a threshold level of jet momentum, is a key issue. This could allow scaling of the valve to higher flow rates. Favorable actuation methods are also of interest to identify. Simultaneous actuation of the two jets, with amplitude modulation to create opposing reduced frequency strategies, in order to potentially promote forced reattachment and forced separation ${ }^{22}$, on opposing valve sides, is an interesting proposal for increased valve response. The geometric properties of the valve that govern the timescales involved in the transition of flow from one channel to the other are of particular importance, as this may also have a role in defining the maximum achievable frequency.

\section{Acknowledgments}

The authors would like to thank the staff of the Enflo Laboratory for help with the experimental setup and acknowledge the work of final year student Wing Lau for aiding with the experimental measurements.

\section{References}

\footnotetext{
${ }^{1}$ Wassermann, F. et al, "Phase-locked 3D3C-MRV measurements in a bi-stable fluidic oscillator", Experiments in Fluids, Vol.54 No.3, 2013, p.1487

${ }^{2}$ Arwatz, G., Fono, I. \& Seifert, A., "Suction and Oscillatory Blowing Actuator," IUTAM Symposium on Flow Control and $M E M S$, Proceedings of the IUTAM Symposium held at the Royal Geographical Society, 19-22 September 2006, Morrison, J. et al (Eds,) Springer, Dordrecht.

${ }^{3}$ Gokoglu, SA., Kuczmarski, MA., Culley, DE., Raghu, S., "Numerical studies of a fluidic diverter for flow control". In Proceedings of 39th AIAA Fluid Dynamics Conf. AIAA-2009-4012 (2009)

${ }^{4}$ Cerretelli, C., Wuerz, W. \& Gharaibah. E., "Unsteady Separation Control on Wind Turbine Blades Using Fluidic Oscillators", AIAA Journal, Vol.48, No.7, 2010, pp 1302-1311

${ }^{5}$ Tesar̆, V. "Fluidic valves for variable-configuration gas treatment", Transactions of the Institution of Chemical Engineers, Part A, Chemical Engineering Research and Design, 83:A9, 2005, p. 1111

${ }^{6}$ Tesar̆, V. \& Bandalusena, H. C. H., "Bistable diverter valve in microfluidics," Experiments in Fluids, Vol.50, No.5, 2010, pp. $1225-1233$

${ }^{7}$ Tesař, V. "No-moving-part valve for automatic flow switching", Chemical Engineering Journal, Vol.162, No.1, 2010, pp. $278-295$

${ }^{8}$ Tesař, V. Hung, C. H. \& Zimmerman, W. B., "No-moving-part hybrid-synthetic jet actuator," Sensors and Actuators A: Physical, Vol:125, No:2, 2006, pp. 159-169

${ }^{9}$ Schatzman, D., Wilson, J., Arad, E., Seifert, A. \& Shtendel, T. "Flow Physics of Drag Reduction Mechanism using Suction and Pulsed Blowing", In Proceedings of 51st AIAA Aerospace Sciences Meeting, AIAA 2013-0488, 2013

${ }^{10}$ Gregory J. W., Gnanamanickam, E. P., Sullivan, J. P., Raghu, S. \& Fellow, D., "Variable-Frequency Fluidic Oscillator Driven by Piezoelectric Devices”, In Proceedings of 43rd AIAA Aerospace Sciences Meeting, AIAA 2005-0108, 2005

${ }^{11}$ Chen, R., and Lucas, G., "An Investigation into the Use of Piezo-Fluidic Combined Units as Fuel Injectors for Natural Gas Engines”, SAE Technical Paper 961987, 1996

${ }_{12}$ Greenblatt, D., \& Wygnanski, I. J. "The control of flow separation by periodic excitation," Progress in Aerospace Sciences, Vol.36, No.7, 2000, pp. 487-545

${ }^{13}$ Tesař, V., Zhong, S. \& Rasheed, F., "New Fluidic-Oscillator Concept for Flow-Separation Control", AIAA Journal, Vol.51, No.2, 2012, pp. 397-405

${ }^{14}$ Franck, J. \& Colonius, T. "Effects of Actuation Frequency on Flow Control Applied to a Wall-Mounted Hump," AIAA Journal, Vol.50, No.7, 2012, pp. 1631-1634

${ }^{15}$ Kotapati, R. B., Mittal, R., Marxen, O., Ham, F.,You, D. \& Cattafesta, L, N. "Nonlinear dynamics and synthetic-jet-based control of a canonical separated flow," Journal of Fluid Mechanics, Vol.654, 2010, pp. 65-97

${ }^{16}$ Smith, B. L. \& Glezer, A. "The formation and evolution of synthetic jets," Physics of Fluids, Vol.10, No.9, 1998, p. 2281
} 
${ }^{17}$ Tesař, V., "Mechanism of pressure recovery in jet-type actuators," Sensors and Actuators A: Physical, Vol:152, No:2, 2009 , pp. 182-191

18 Martin, N. D., "Preliminary Experimental Results of Synthetic Jet Flow Control Over a NACA 0015," in Joint ERCOFTAC/PLASMAERO Workshop, Toulouse, France, December 2012, pp. 36-38.

${ }^{19}$ Persoons, T., "General Reduced-Order Model to Design and Operate Synthetic Jet Actuators," AIAA Journal, Vol.50, No.4, 2012, pp. 916-927

${ }^{20}$ Gallas, Q., Holman, R., Nishida, T., Carroll, B., Sheplak, M., \& Cattafesta, L. "Lumped element modeling of piezoelectricdriven synthetic jet actuators," AIAA Journal, Vol.41, No.2, 2003, pp. 240-247

${ }^{21}$ Jabbal, M., Liddle, S., Potts, J. \& Crowther, W., "Development of design methodology for a synthetic jet actuator array for flow separation control applications", Proceedings of the Institution of Mechanical Engineers, Part G: Journal of Aerospace Engineering, Vol.227 No.1, 2013, pp. 110-124

${ }^{22}$ Darabi, A., \& Wygnanski, I. "Active management of naturally separated flow over a solid surface. Part 2. The separation process", Journal of Fluid Mechanics, Vol.510, 2004, pp. 131-144 